\title{
Development of Superhydrophobic Surface for Medical Textiles
}

\author{
Youn-Soo Shim 1 , So-Young Park² and Myoung-Hee Shim ${ }^{3 *}$ \\ 'Department of Dental Hygiene, College of Health Science, Sunmoon University, Asan-si, Korea; \\ ysshim@sunmoon.ac.kr \\ ${ }^{2}$ Department of Preventive and Public Health Dentistry, College of Dentistry, Wonkwang University, Iksan-si, Korea; \\ 1101so-young@hanmail.net \\ ${ }^{3}$ Textile and Fashion R\&D Center, Korea Textile Trade Association, Seoul, Korea; textra@hanmail.net
}

\begin{abstract}
The objective of this study was to fabricate superhydrophobic surface with superhydrophobic property using nanoparticles and water-repellent agents for medical textiles. The wettability of the super hydrophobic surface was compared to that of other materials having a variety of roughness and surface energy. Microstructures were fabricated with 100\% PET textiles having various yarn thickness and yarn finishing. Nano-sized structures were fabricated by finishing the PET textiles with CNTs nanoparticles using water-repellent agent. Contact angle (CA), contact angle hysteresis and shedding angle analysis were evaluated by Theta Lite contact angle meter (attension, Finland). Also, breathable waterproof properties were measured by water vapor transmission rate (ASTM 96- $\mathrm{CaCl}_{2}$ ) and air permeability (ISO 9237-1995). The results indicate that the super hydrophobicity was strongly influenced by the binary structure having nano-scaled roughness than by the chemical composition of surface. It was proven that the shedding angle, the angle at which a water droplet rolls off the surface, was reasonable method to analysis the super hydrophobic properties. Also, it was shown that all of the water-repellent agents and nano-particles did not decrease air permeability and water vapor transmission rate noticeably. The DTY sample treated with CNTs exhibited the highest super hydrophobic properties, having static contact angle of $160^{\circ}$ and shedding angle of $4.4^{\circ}$. These results indicated that the long nanofiber shape of carbon nano tubes successfully maintained the trapped micro sized air layers like a papillae of lotus leaf.
\end{abstract}

Keywords: Medical Textile, Nano Particle, Superhydrophobic, Self-Cleaning

\section{Introduction}

Self-cleaning surfaces, for their convenience in less soiling and less need of washing, have drawn much attention. Especially, medical textiles need superhydrophobicity to protect infection by pathogenic fungus. Among the methods to develop the self-cleaning textiles, fabrication of textiles surface having the binary structure like lotus leaf surface for an easy removal of contaminants has recently been of particular interest. Lotus leaf is a typical super hydrophobic surface with self-cleaning function and surface has binary structure with tensof-nano sized particles stuck to tens-of-micro sized protuberances and composed of wax has low surface energy. Wax ingredient of Lotus leaf itself has $110^{\circ}$ contact angle but has hysteresis of high static contact angle above $160^{\circ}$ and low sliding contact angle of $2^{\circ}$ by dual structure of surface. Thus these surfaces have properties such as antisticking, anti-contamination, and self-cleaning are expected. These properties are desirable for many industrial and biological applications such as antibiofouling paints for boats, antisticking of snow for antennas and windows, self-cleaning windshields for automobiles, metal refining, stain resistant textiles, antisoiling architectural coatings ${ }^{1}$.

The wettability on a rough surface in terms of contact angle of liquid can be described by the Cassie-Baxter model (Equation 1$)^{2}$ :

$$
\cos \theta_{\gamma}^{C B}=f_{1} \cos \theta_{e}+f_{2} \cos \theta_{\dot{e}}
$$

*Author for correspondencev 
$\theta_{r}^{C B}$ : apparent contact angle of liquid on a rough surface in a Cassie-Baxter state

$\theta_{e}$ : equilibrium contact angle when the liquid droplet sits on a smooth surface 1

$\theta_{e^{\prime}:}$ equilibrium contact angle when the liquid droplet sits on a smooth surface 2

$f_{1:} \quad$ fraction of surface 1 area in contact with liquid

$f_{2}$ : fraction of surface 2 area in contact with liquid

In the earlier report in our study ${ }^{3}$, theoretical validation of the Cassie-Baxter model was provided on the hydrophobic woven polyester fabrics, with the estimation of fabric roughness represented by $f_{1}+f_{2}$. The results shown that theoretical contact angle values of $20 \mathrm{~F}$ and $50 \mathrm{~F}$ than those for DTY fabrics. And the samples made of 50 denier were investigated by optimal specification for superhydrophobic fabric.

Especially, textile products polluted by pathogenic microorganism play the role of medium causing transmitted disease. Surgical gown, bed sheet in a hospital room, nurse uniform deliver virus of patient to other place and they are becoming the cause of nosocomial infection. Therefore, utilizing superhydrophobic textile is suggested to minimize virus transmission by patient's blood and physical secretion.

The wettability of liquids on solid surfaces can be affected by the properties of liquids. First, increased temperature of liquid, the surface tension would be decreased. Therefore under assumption that surface tension of solid-air interface not being changed, increased temperature of water, surface tension of solid-liquid and liquid-air would be decreased ${ }^{4}$. As a result, contact angle would be decreased. Relation liquid's temperature and surface tension could be verified through below formula (Equation 2).

$$
\gamma=75.714-0.1414 t-0.25399 \times 10^{-3} t^{2}
$$

$\gamma$ : surface tension of water at temperature

t: temperature of water droplet

This means on flat surfaces high temperature liquids wet the surface easier than low temperature liquid. When the temperature of water is increased, the surface tension of water decreases. Hot water molecules wet the solid surface easier than cool water. The air bubbles trapped in the cavities escaped away from the rough surface due to the heat from the hot water.

Also, the contact angle and sliding angle can be affected by the size or weight of water. It is reported that static contact angle and sliding angle is being decreased as weight of liquid being increased. Wolfram and co-authors have proposed an empirical equation describing the sliding angle of droplets on flat surfaces of various materials ${ }^{6}$.

$$
\sin \pm=\mathrm{k} \frac{2 r \pi}{m g}
$$

Where $\alpha$ is the sliding angle, $\gamma$ is the radius of the contact circle, $m$ is the weight of the droplet, $g$ is the gravitational acceleration, and $k$ is a proportionality constant.

The body should be protected from harmful foreign substances with using textiles having high water repellent property because hospital has circumstances that patients and doctors are easily exposed to diverse secretion, blood and liquid medicine.

Therefore, the purpose of this study is to develop the superhydrophobic surface using nanoparticles and water repellent agent for medical textiles. In this study, a superhydrophobic fabric mimicking the binary rough structure of lotus leaf was fabricated employing CNT and fluoro chemicals, and the effect of CNT concentration and comfort of the finished fabrics was investigated.

\section{Experimental}

\subsection{Materials and Methods}

Microstructures were prepared with 100\% Polyester woven fabrics which is optimal substrate inthe earlier report.The number given in the specimen code indicates the denier of the yarn, and the following alphabet code $\mathrm{D}$ is the Drawn Textured Yarn (DTY) finishing. The characteristics of the fabric are presented in Table 1.

In this study, Carbon Nanotube (CNT) was used with functional groups containing oxygen such as $-\mathrm{COOH}$ stuck to as treated CNT with strong acid through Figure 1. Type of CNT is tens-of-micro sized length with 10 20nm diameter.

Water repellent agents is used with diluting to solvent, to produce Teflon AF composed of PDD-TFE to $0.6 \mathrm{vol}$

Table 1. Characteristics Of The Fabric Specimens

\begin{tabular}{|c|c|c|c|c|}
\hline \multirow{2}{*}{ Code } & \multicolumn{4}{|c|}{ Physical properties } \\
\cline { 2 - 5 } & Yarn count & $\begin{array}{c}\text { Weight } \\
\left(\mathrm{g} / \mathbf{m}^{2}\right)\end{array}$ & $\begin{array}{c}\text { Thickness } \\
(\mathbf{m m})\end{array}$ & $\begin{array}{c}\text { Density } \\
\text { (in cm) }\end{array}$ \\
\hline 50D & $\begin{array}{c}50 \text { denier yarn in } \\
72 \text { filament DTY }\end{array}$ & 65.53 & 0.13 & $69 \times 44$ \\
\hline
\end{tabular}



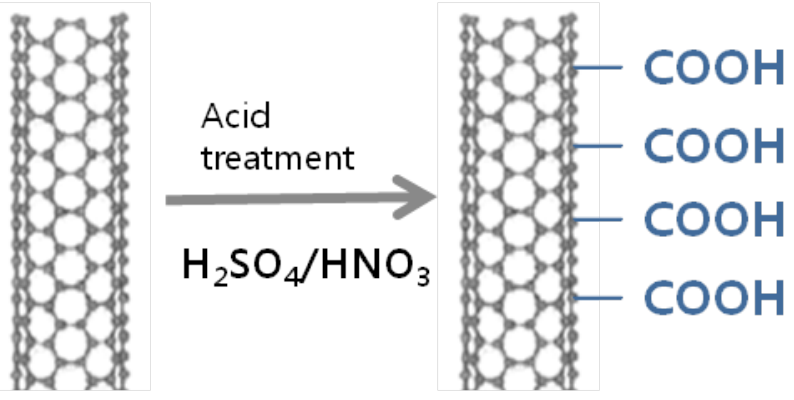

Figure 1. Surface of CNT treated with strong acid.

$\%$ density, used with diluting to FC-75(3M) composed of Perfluoro-2-butyltetrahydro-furan, monomer. CNT of $1 \mathrm{wt} \%$ treated with acid was put to this agent and used with treatment of ultrasonic waves for $20 \mathrm{~min}$ to improve dispersibility with finishing agent.

Putting $1 \ell$ of complex finishing agent dispersed CNT to bath of padding machine then dipping fabric by $25 \mathrm{~cm}$ $X 25 \mathrm{~cm}$ and push through roller of 50psi. And dry and curing was processed.

For producing specimen of differing density of CNT, it was done in a way of repetitive dipping-padding process.

\subsection{Analysis}

Before and after processing, Fe-SEM (Supra 55VP, Germany) was used to analyze surface characteristic of specimen's appearance and surface roughness. Also AFM (Atomic force microscope) was used to analyze nano-sized surface roughness by finishing agents and image size was measured as non-contact mode of $10 \mu \mathrm{m} \times 10 \mu \mathrm{m}$. To evaluate super hydrophobic, we measured static contact angle and shedding angle value using Theta Lite (Attension, KSV Instruments).

The physical property related to comfort of processed sample was measured with water vapor transmission rate (ASTM E 96) and air permeability (ASTM D 737).

\section{Results}

As a result of authentication by Figure 2., specimen processed of dipping-padding being CNT dispersed to Teflon AF has minute surface roughness with CNT spread to fabric surface irregularly.

We analyzed Fe-SEM and AFM image to compare surface roughness of two types' samples have different concentration of CNT. On comparing a difference in appearance affected by CNT's density being attached to

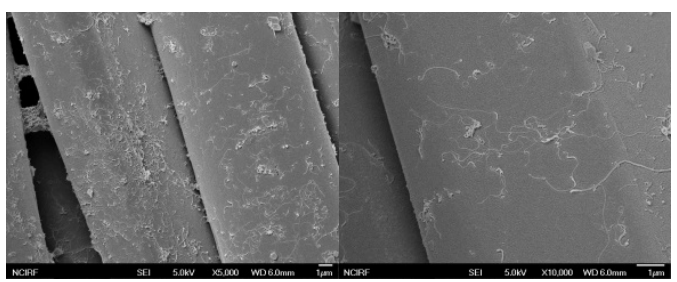

3.1.1.1 Fluoro-C1

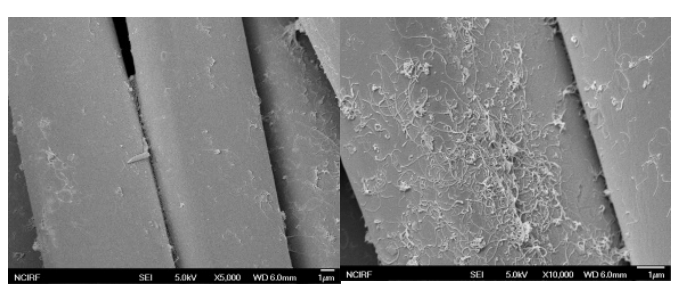

3.1.1.2 Fluoro-C2

Figure 2. (a) Fe-SEM images $(\times 5,000, \times 10,000)$ of the $50 \mathrm{D}$ treated with low concentration. (b) and high concentration.

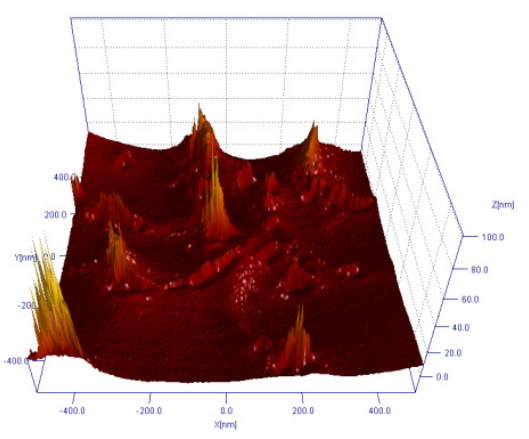

(a)

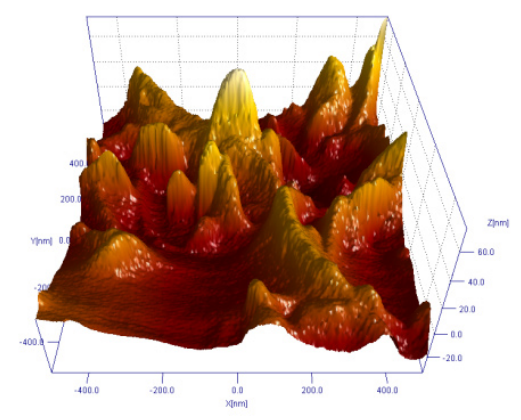

(b)

Figure 3. Difference of AFM images by concentration of CNTs. (a) Low concentration (Fluoro-C1), (b) High concentration (Fluoro-C2). 
50D sample's textile based on Figure 1., we can see that Fluoro-C1 had process of dipping-padding is more little in $\mathrm{CNT}$ attaching than Fluoro-C2. And we can see the difference of roughness of CNT through Figure 2., AFM as well. In this study, we can control concentration of nano particles with repeating dipping-padding but distributing equally to the surface of textile is restricted. Therefore, it is required of suitable proposal in treatment method that could distribute CNT agent to textile surface.

To investigate the effect of CNT concentration on the hydrophobicity of treated fabrics, CNT was treated on $50 \mathrm{D}$ one time and two times by dip-pad processes, and coded as Fluoro-C1 and Fluoro-C2 respectively.

Static contact angle was examined for 50D treated with different CNT concentrations in Figure 4., and the differences among the specimens were not distinct. However, shedding angle measurements presented in Figure 5. shows the observable tendency of the level of hydrophobicity, decrease of shedding angle with higher level of CNT treatments, not to mention the difference between the untreated and treated specimens. The

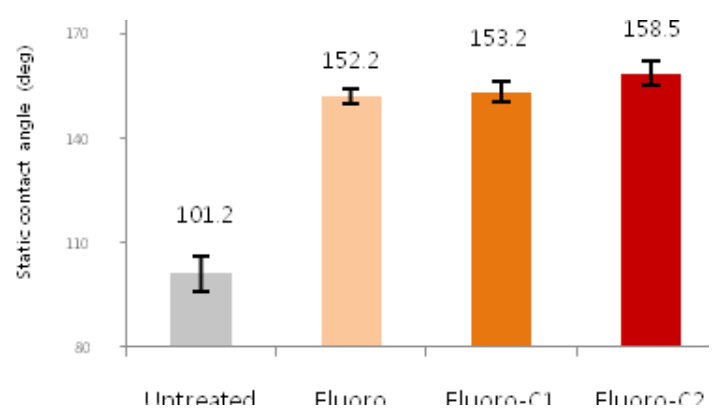

Figure 4. Effect of nano-particle addition on static contact angles.

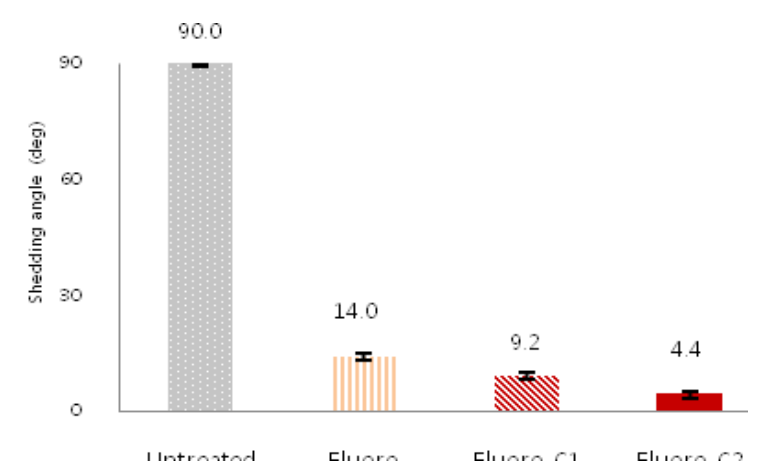

Figure 5. Effect of nano-particle addition on shedding angles. nano-sized roughness contributed by CNT addition on fiber surface was speculated to result in decreased shedding angle than Fluoro only treated specimen, and the higher density of CNT on fiber surface would have produced roughness in higher density. It was deduced that the nano-sized roughness generated by carbon nano tubes, and in rather densely distribution, was more effective in holding air traps under nano-scale bumps and in reducing the fabric surface area in contact with water droplet.

From the experimental results, it was observed that shedding angle, rather than static contact angle, is more relevant representation to tell the level of hydrophobicity for a superhydrophobic material whose static contact angle close to or higher than $150^{\circ}$. By this result, the surface roughness by nano-sized particles has a tendency that the more this particles, the higher super hydrophobic. Particularly, a longitudinal waves nano-particles such as CNT enhance surface roughness and for this, we presume that contact area of water drop and surface area is decreased.

This bouncing phenomenon, closely associated with self-cleaning property, was clearly observed only from the specimen Fluoro-C2 in this study, which demonstrates the feasibility of self-cleaning application of this fabric material. Low surface energy attributed by Fluoro coating and roughness contributed by $\mathrm{CNT}$ addition would have resulted in easier bouncing of a droplet by reducing the interface between the substrate and liquid and lowering the surface energy of the textile. Figure 6. shows the water droplets placed on a substrate $50 \mathrm{D}$ treated with Fluoro-C2, in the volume of $3.6 \pm 0.2 \mu l$ and $12.5 \pm 0.1 \mu l$.

To apply these superhydrophobic fabrics as hospital gown and bed sheet was evaluated water vapor transmission rate and air permeabilityto comfort. In Figure 7., it was shown that all of the water-repellent agents and nano-particles did not decrease water vapor transmission rate noticeably. In Figure 8., the air permeability was shown that a slight decreased values by concentration of CNT. But the values are enough to transmit heat and moisture.

Figure 9. is the Fe-sem images of samples without and with CNT nano-particles. The finish agent treated CNT didn't obstruct microporous between the fiber and fiber. This being so, these microporous can maintain outstanding water vapor permeability and air permeability. 


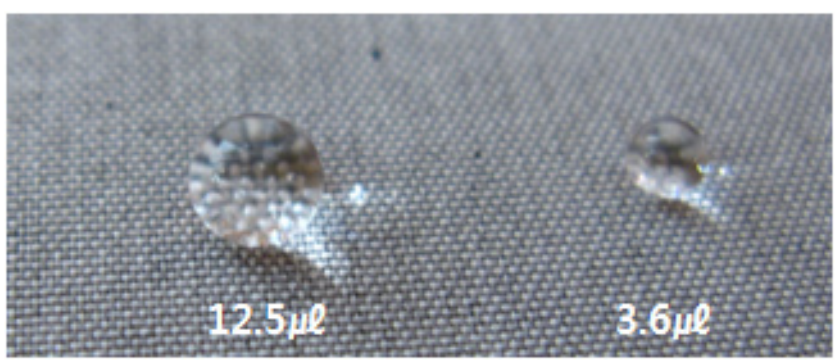

Figure 6. Photograph image of water droplets on a 50D sample treated with Fluoro-C2.

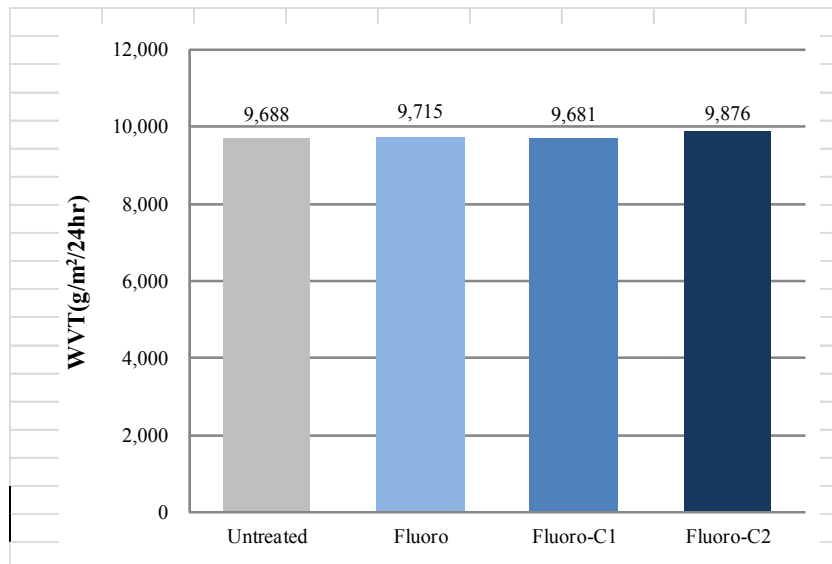

Figure 7. Water vapor transmission of the specimens (50D) treated with water-repellent agents.

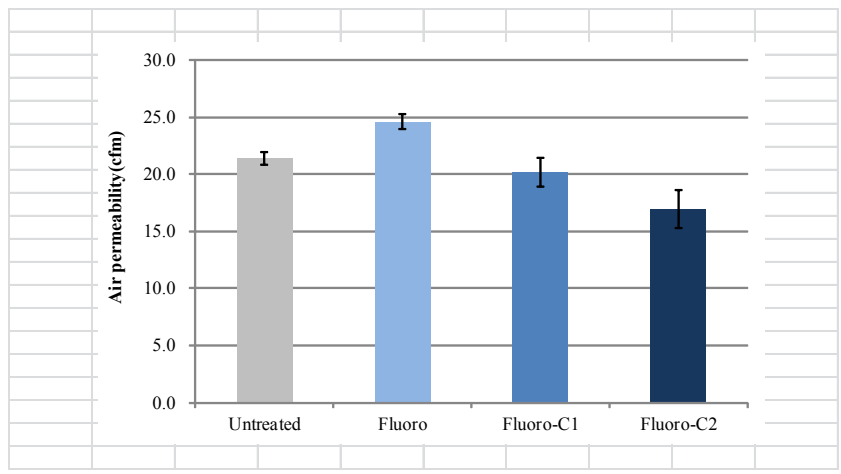

Figure 8. Air permeability of the specimens (50D) treated with various water-repellent agents.

\section{Conclusion}

The functional textiles such as super hydrophobic fabrics can be used as barrier to prevent from harmful substance, blood and diverse secretion. In this study, super hydrophobic textiles having micro-nano rough surface like lotus leaf was prepared with polyester woven fabrics which were treated with CNTs dispersed in water-repellent agents. Especially, the super hydrophobicity of the treated fabric increase with the increase of concentration of CNT nano-particles. But this finish maintained outstanding water vapor permeability and air permeability.

As a result, the 50D treated with Teflon AF-CNTs exhibited the superhydrophobic properties, having static contact angle $>150^{\circ}$, sliding angle $<10$. These results disclosed that the carbon nano tubes with long nanofiber shape successfully maintained the trapped air layers like a papillae of lotus leaf.

\section{References}

1. Li X, Reinhoudt D, Crego-Calama M. What do we need for a superhydrophobic surface? A review on the recent progress in the preparation of superhydrophobic surfaces. Chemical Society Reviews. 2007; 36:1350-68.

2. Michielsen S, Lee HJ. Design of a superhydrophobic surface using woven structures. Langmuir. 2007; 23:6004-10.

3. Shim MH, Kim J, Park CH. The effects of surface energy and roughness on the hydrophobicity of woven fabrics. Textile Research Journal. 2014; 84:1268-78.

4. Liu Y, Chen X, Xin JH. Can superhydrophobic surfaces repel hot water? Journal of Materials Chemistry. 2009; 19:5602-11.

5. Nosonovsky M, Bhushan B. Multiscale dissipative mechanisms and hierarchical surfaces. Berlin, Heidelberg: Springer; 2008. p. 9.

6. Buzagh A, Wolfram E, Kolloid Z, Wolfram E, Faust R. Wetting, Spreading and Adhesion. In: Padday JF, editor. London: Academic Press; 1978. p. 125. 